\title{
Optical characterisation of hydroxide catalysed bonds applied to phosphate glass
}

Grégoire Lacaille, Valentina Mangano, Anna-Maria A. van Veggel, Christian J. Killow, Peter E. MacKay, et al.

Grégoire Lacaille, Valentina Mangano, Anna-Maria A. van Veggel, Christian J. Killow, Peter E. MacKay, Sheila Rowan, James Hough, "Optical characterisation of hydroxide catalysed bonds applied to phosphate glass," Proc. SPIE 10448, Optifab 2017, 1044825 (16 October 2017); doi: $10.1117 / 12.2279693$

\section{SPIE. Event: SPIE Optifab, 2017, Rochester, New York, United States}




\title{
Optical characterisation of hydroxide catalysed bonds applied to phosphate glass
}

\author{
Grégoire Lacaille* $^{* a, b}$, Valentina Mangano ${ }^{\mathrm{b}}$, Anna-Maria A. van Veggel ${ }^{\mathrm{b}}$, Christian J. Killow ${ }^{\mathrm{b}}$, \\ Peter E. MacKay ${ }^{\mathrm{a}}$, Sheila Rowan ${ }^{\mathrm{b}}$, James Hough ${ }^{\mathrm{b}}$ \\ ${ }^{a}$ Gooch \& Housego, Dowlish Ford, TA19 OPF, Ilminster, UK; \\ ${ }^{\mathrm{b}}$ SUPA, Institute for Gravitational Research, School of Physics and Astronomy, University of \\ Glasgow, G12 8QQ, Glasgow, UK
}

\begin{abstract}
We apply the Hydroxide Catalysis Bonding ( $\mathrm{HCB}$ ) technique to phosphate glass and measure the reflectivity and Light Induced Damage Threshold (LITD) of the newly formed interface. HCB is a room temperature, high performing process which was designed for astronomical research glass assemblies and played a key role in the detection of gravitational waves, a breakthrough in contemporary science. The bonds have numerous assets including mechanical strength, stability, no outgassing and resistance to contamination which are of high interest in the precision optics industry. However only little research has been done on their optical properties and mostly on silica based materials. In this paper, we use HCB to bond phosphate glass at room temperature with the goal of designing composite components for solid state laser gain media. We change the solution parameters to identify how they influence the final properties of the bonds: the LIDT at $1535 \mathrm{~nm}$ in long pulse regime and the reflectivity at $532 \mathrm{~nm}$ are investigated. The measurement of the incidence dependent reflectance allows estimating the thickness and refractive index of the bond in a non destructive process. The best performing set of parameters yields a LIDT of $1.6 \mathrm{GW} / \mathrm{cm}^{2}\left(16 \mathrm{~J} / \mathrm{cm}^{2}\right)$ and a reflectivity below $0.03 \%$ which makes it suitable for use in high power lasers. The bond thickness is derived both from Scanning Electron Microscopy and the reflectivity measurements and is in the range of 50-150 nm depending on the parameters. Finally, the bonds survive cutting and polishing which is promising for manufacturing purpose.
\end{abstract}

Keywords: Room temperature bonding, Phosphate glass, Light induced damage, Reflectivity, Bond thickness.

\section{INTRODUCTION}

Hydroxide Catalysed Bonding (HCB) was developed by Gwo during the construction of Gravity Probe B in $1998^{1}$. The technique offers numerous assets for glass assemblies to be launched in space such as mechanical strength, precision alignment as well as impermeable and outgassing-free bonds. All these properties are also sought in the photonics industry where composite components allow the tackling of problems such as thermal lensing by bonding appropriate materials ${ }^{2}$. Some other techniques have been consistently used in the past years for that purpose. Optical contacting is the most straightforward technique but offers poor strength, is sensitive to contamination by water vapour and thus often requires sealing and leaves no room for alignment once the pieces are put in contact ${ }^{3}$. Diffusion Bonding adds an additional heating treatment to optical contacting to successfully solve the strength and contamination problem ${ }^{4}$. However the heat treatment may induce thermal stresses or limit the range of usable materials due to thermal expansion and the difficulties in alignment can only be solved using expensive setups. Adhesive bonding requires less stringent surface preparation as the adhesive layer will make up for the roughness of the surface but degassing and average precision in alignment limit the range of applications ${ }^{5}$. Finally, glass frit bonding is limited to materials within a certain range of coefficients of thermal extension and has the same alignment issue as adhesive bonding coming from the viscous and relatively thick intermediate layer ${ }^{6}$. $\mathrm{HCB}$, although outclassed in certain aspects by these techniques, has

Optifab 2017, edited by Julie L. Bentley, Sebastian Stoebenau, Proc. of SPIE Vol. 10448, $1044825 \cdot$ (c) 2017 SPIE · CCC code: 0277-786X/17/\$18 · doi: 10.1117/12.2279693 
been proven to offer a good compromise of properties and validated as a reference bonding technique for glass assemblies in recent projects ${ }^{7}$. However, investigations on the optical properties of bonds made using this technique are still in their infancy and therefore more studies need to be carried before they can be used in photonics components. Two properties of the new interface due to the bond are particularly of interest: the Light Induced Damage Threshold (LIDT) and the reflectivity. The LIDT test requires measuring the maximal optical power that an interface can withstand without being permanently damaged and therefore is a mandatory step before putting an optical component into its operating position. Interface reflectivity is a source of reflected beams which may interfere with the main one and degrade its quality while also causing loss in transmission. Therefore low reflectivity is required when introducing new interfaces on the beam path. It usually comes from the small voids left from the bonding (surface mismatch or porosity of the bond) or directly from the refractive index mismatch between the bond and the bonded pieces due to different chemistry.

Pioneering work on both properties was carried by Sinha who explored them for applications in termination of fused silica fibres ${ }^{8}$. He studied the influence of the parameters of the solution used and the heat treatment applied. Subsequent works studied one or the other of these properties for different purposes ${ }^{9,10,11}$. The common point of these studies is their focus on fused silica for its good properties to build assemblies (low mechanical loss at ambient temperature, low thermal expansion coefficient). Yet, when moving towards the photonic industry a new range of properties may be required and fused silica may get replaced by better (or differently) performing materials. Phosphate glass for example is preferred as an amorphous glass for solid state laser gain media ${ }^{12,13}$. It offers better transition cross sections and a higher ceiling for the doping rate which broaden the reachable range of laser parameters. It also has a negative thermo-optic coefficient which can compensate the usual positive ones of the other components and therefore lower the total thermal lens of the whole setup. However the difference in the composition and thus optical properties of the materials (refractive index of about 1.56 for phosphate glass compared to 1.48 for fused silica) gives rise to concerns about whether the conclusions of the previous works are transferrable or not.

This paper reports on the use of $\mathrm{HCB}$ and silicate bonding, which is a derived technique (see section 2), to join phosphate glass components. The objective is to combine the interesting properties of the material and the ones of the bond to design a new range of highly performing components such as composite laser gain media. The LIDT at $1535 \mathrm{~nm}$ and reflectivity of the bond are measured for different solutions used and different curing time in the case of reflectivity. The samples are then cut and polished which assesses the mechanical strength of the bond and makes observation through Scanning Electron Microscopy (SEM) possible.

\section{HYDROXIDE CATALYSED BONDING}

HCB uses a hydroxide solution to etch the surface of amorphous glass into a gel which then dehydrates to join the two components. For most efficient bonding the surfaces should be flat to $60 \mathrm{~nm}$ peak to valley and made hydrophilic by a surface activation process ${ }^{11}$. A drop of solution is laid on one surface and will spread widely if the surface is properly activated. The two surfaces are then brought in close contact and the surface etching starts to occur, causing the $\mathrm{pH}$ of the solution to drop. Once a threshold $\mathrm{pH}$ is reached (11 in the case of silica glass), the gel polymerises and dehydrates leaving the two surfaces bonded by a rigid network as showed in Figure 1.

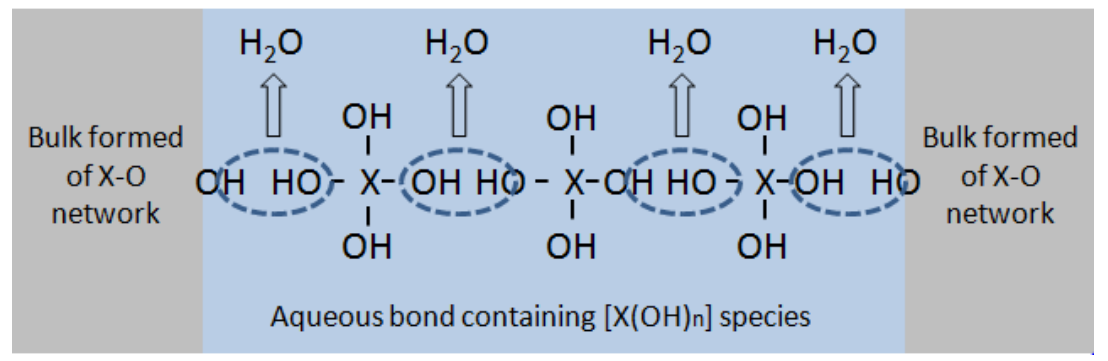

Figure 1: Bridging between two surfaces by dehydration and polymerisation of the bonding solution. $\mathrm{X}$ represents atoms that can be etched from the bulk or already present in the solution. In this study it will be either phosphate P (from the bulk) or silicon Si (from the solution). 
The process has been widely studied and accepted for amorphous silica based materials which allow the formation of dense fused silica networks between the surfaces. For crystalline oxides (such as quartz or sapphire) which are not as easy to etch, a sodium silicate solution (sodium hydroxide solution with silicates added to it) can be used to increase the amount of silicate ions forming the bridging network, improving the strength of the bond ${ }^{14}$. This may be an issue when considering the optical properties of the interface as the different refractive index of the bond material can induce undesired reflectivity if the materials to be bonded have a different refractive index from silicon dioxide.

Investigations on the chemistry of phosphate glass suggest that a similar process can occur using a sodium hydroxide solution to dissolve phosphate chains which will bond the two surfaces upon polymerisation and dehydration ${ }^{15}$. The material being an oxide, it is also expected to be possible to form a silicate network if a sodium silicate solution is used instead of sodium hydroxide. The resulting bond probably mixes dissolved phosphate and siloxane chains although chemical analysis has not be carried out. Both sodium hydroxide and sodium silicate solutions will be used in this work and the performances of the different bonds will be compared.

\section{METHODS}

\subsection{Bonding}

The phosphate glass material used is a EYP8 glass from Glass Technology Services. Samples were manufactured at Gooch and Housego (Ilminster, UK). Discs are $\varnothing 30 \mathrm{~mm}$ x $3 \mathrm{~mm}$ and surfaces are polished to $60 \mathrm{~nm}$ peak to valley, which is checked using a Zygo GPI XP/D interferometer, before a chemical-mechanical finishing with a Kemet Col-k slurry and cleaning in an acetone bath. Surfaces are cleaned again with spectroscopic grade methanol immediately before bonding. A first set of samples is prepared, three samples using a 0.01M sodium hydroxide solution (S2770 Sigma diluted 100 times) and the three other a sodium silicate solution (Sigma Aldrich, which is $14 \%$ sodium hydroxide, $27 \%$ silicate and $59 \%$ water in volume) diluted to 1:6 parts in volume in distilled water. An amount of $1 \mu 1 / \mathrm{cm}^{2}$ is laid on one surface with a micropipette and the second part is then brought into contact. The potential bubbles are chased towards the edges by gently moving the two parts which are aligned before the bond starts gripping. Samples are then left for curing at room temperature and ambient atmosphere until tested. In the following, HCB (Hydroxide Catalysed Bond-ed) refers to the samples bonded using a hydroxide solution while SB (Silicate Bond-ed) refers to the samples bonded with sodium silicate solution.

An additional set of samples was bonded with the purpose of seeing the influence of the solution concentration of the sodium silicate solution on the tested parameters. Samples are $35 \times 10 \times 5 \mathrm{~mm}$ blocks and are bonded on the largest face. The solutions used are sodium silicate solution diluted to 1:3, 1:6 and 1:10 parts in volume in distilled water. The deposited volume is $0.8 \mu 1 / \mathrm{cm}^{2}$. The samples of this set will be referred as SB3, SB6 and SB10 (Silicate Bond-ed and dilution indicator) in the following. A few parameters changed between the two sets which will prevent from directly comparing them. First, it appeared that the glass used for the first set was of lower optical quality than for the second set. Also the geometry was changed to better fit the requirements of the reflectivity setup. Finally, the volume of solution was modified to get in line with other studies conducted at the time on different materials. However, both sets still bring novel results and as the tests are made in similar conditions they will be carefully compared when deemed relevant.

\subsection{Light induced damage threshold}

LIDT tests were carried out at Belford research Ltd (Edinburgh, UK). A sample of each type from the first set is tested (HCB and SB) after 120 days of curing and the samples from the second set (SB3, SB6, SB10) after 46 days. The wavelength of interest is $1535 \mathrm{~nm}$ as it is the main emitting wavelength of the glass used (Erbium doped phosphate glass - as a lasing medium). The power is increased incrementally at each step. For each power, ten sites are probed with the beam focused on the bond and the number of sites damaged is noted. Increasing the power should increase the probability of damaging the sample and the LIDT is taken as the average between the highest power not leading to 
damage and the first power for which damage is witnessed. The complete test specifications and an illustration of the process are displayed in Figure 2.

\begin{tabular}{|c|c|c|c|}
\hline \multicolumn{2}{|c|}{ TEST SPECIFICATIONS } & \multirow{21}{*}{$\begin{array}{c}\text { Increasing } \\
\text { power }\end{array}$} & \\
\hline Test & Laser Induced Damage Threshold & & \\
\hline LIDT Type & S-on-1 & & \\
\hline Sample Size & $25.4 \mathrm{~mm} \times 6-6.5 \mathrm{~mm}$ & & \\
\hline Optic or Witness & Optic & & \\
\hline Side to Test & Interface & & \\
\hline Polarization & Linear & & \\
\hline Wavelength $(\mathrm{nm})$ & 1535 & & \\
\hline Angle of Incidence (deg) & $0^{\circ}$ & & \\
\hline Spot Diameter $\left(1 / \mathrm{e}^{2}\right)(\mu \mathrm{m})$ & Smallest Spot 200 & & \\
\hline Pulse Duration (FWHM) (ns) & 10 & & \\
\hline Repetition Rate $(\mathrm{Hz})$ & 10 & & \\
\hline Threshold Determination & Least Fluence & & \\
\hline Test Beam Profile & TEM $_{\mathrm{oo}}$ & & \\
\hline Axial Modes & Multiple & & \\
\hline Number of Sites per Row & 10 & & Non Damaged \\
\hline Number of Shots per Site & 20 & & \\
\hline Number of Rows tested & 20 & & \\
\hline Total Number of Shots & 4000 & & \\
\hline Sample Handling & Cleanroom Glove & & \\
\hline Test Compliance & $\begin{array}{c}\text { ISO Standards } 21254-1 ; 21254-2 ; 21254- \\
3 \text { and ISO-TR-21254-4 } 2011 \\
\end{array}$ & & \\
\hline
\end{tabular}

Figure 2: Left: LIDT test parameters. Right: Layout and illustration of the process. The disk represents the bond interface of a sample that is probed at different locations, increasing the power at each row. The laser is focused on the bond and the bonded parts are hidden for clarity. The spread damage illustrates the fact that the sample will not be consistently damaged before the power is largely above the power leading to the first damage but the probability of damage increases with the power.

Although the concept of the LIDT measurement is rather simple and straightforward, the underlying mechanisms have been under investigation for years and are still relevant topics in current papers ${ }^{16,17}$. Details of the mechanisms will not be discussed here as it would be beyond the scope of this paper. However it can be noted that the test was made in the regime of long pulses $\left(10^{-8} \mathrm{~s}\right)$ and therefore the main damaging mechanism is attributed to the dielectric breakdown of the material (compared to thermal effects in the continuous regime for example).

\subsection{Reflectivity}

The setup for the reflectivity measurements was built and refined at the Institute of Gravitational Research of the University of Glasgow ${ }^{9}$. The layout is presented in Figure 3.

A continuous beam is shot on the sample where it reflects on the front, bond and back surfaces to a photodetector (PD2). Two wavelengths $(532 \mathrm{~nm}$ and $633 \mathrm{~nm}$ ) can be selected and, as unfortunately infrared wavelength was not available, the green laser $(532 \mathrm{~nm})$ was used for the experiment. The beam goes through a spatial filter and can be polarised parallel (P-pol) or perpendicular (S-pol) to the plan of incidence thanks to a polarising beam splitter (PBS) and two rotating half wave plates $(\lambda / 2)$. The light is focussed on the bond with an additional lens. The sample is placed on a rotating stage so that the angle of incidence can be varied. By masking photodiode (PD2) with a thin slit and translating it, it is possible to isolate the bond spot from the much brighter surface reflections. The stage displacement and the acquisition are automated through a Labview program. The reflectance of the bond is measured for each polarisation by taking the ratio between the incident power (recorded by PD1) and the reflected power from the bond (recorded by PD2) after calibration of both detectors. Both signals go through a lock-in amplifier to lower the noise in the measurement. The incident power is read and noted before and after each measurement in order to account for a potential drift of the power, the measurement taking several hours for each sample. 


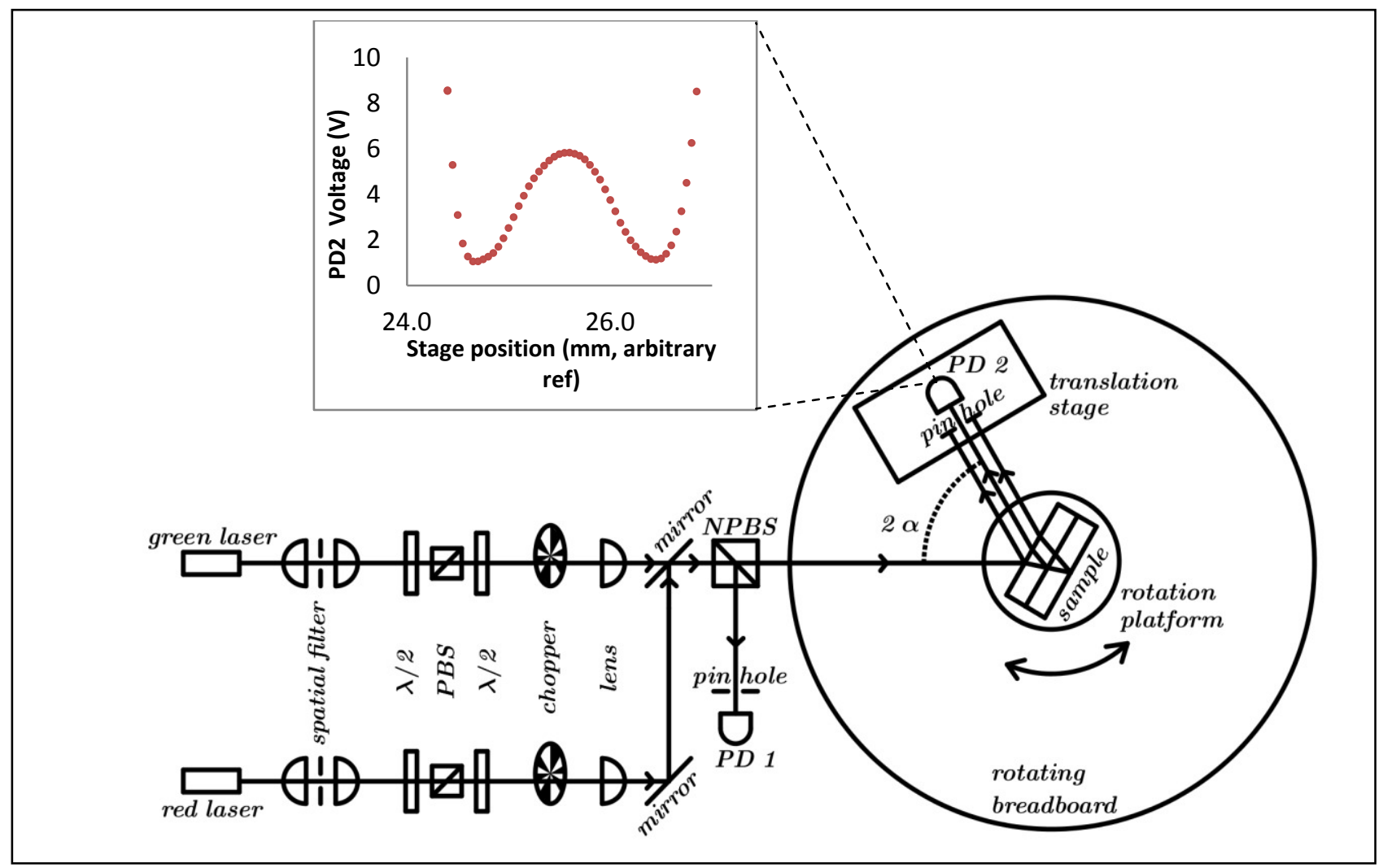

Figure 3: Optical Layout of the reflectivity measurement setup. The data acquisition circuit is not shown to simplify the drawing. The graph shows a typical voltage profile measured by the photodiode PD2 when the stage is translated. The bond spot stands out from the two surfaces reflections, marked by the high slopes at the boundaries.

An example of a raw measurement of the reflected power (in Volts) when the translation stage is moved is shown in Figure 3. The interval of interest is delimited by the high slopes on the boundaries which correspond to the very bright reflections from the surfaces. The relative error on the reflectance value, which is mostly due to positioning of the detector and the sample, was estimated to $\pm 5 \%$. This procedure is repeated for several angles of incidence (with a $5^{\circ}$ increment). The interval is determined by the spatial resolution of the photodiodes on the one hand and the sample geometry on the other hand. At low angle of incidence, the three reflected beams are close and the bond spot is hidden by the much brighter reflections from the front and back surfaces. At large angles of incidence edge effects occur as the beams hit the side face of the sample.

\subsection{Bond intrinsic properties}

It can be argued that the experimental conditions for the reflectivity measurements are quite far from the ones which may be encountered in commercial applications. The visible wavelength used is not really relevant for the studied material and normal incidence cannot be obtained. However, the angle-dependant reflectivity allows two intrinsic properties of the bond to be obtained: its thickness and its refractive index. This is very convenient as these properties will not depend on the experimental conditions and therefore can be directly used as specifications for products. A MATLAB script is used to browse a grid of refractive index values (between 1.3 and 1.56) and thickness values (between $1 \mathrm{~nm}$ and $2000 \mathrm{~nm}$ ) and calculate the theoretical reflectance for $\mathrm{S}$ and $\mathrm{P}$ polarisation with every couple of values possible. A Bayesian likelihood analysis then allows finding the couple that fits the best the experimental data. More details on the model and method can be found in the referred paper'. 


\section{RESULTS}

\subsection{SEM results}

All samples bonded easily within a few tens of seconds regardless of the solution used. The interface looked clear and mostly free of defects. Only one sample was discarded due to a large fringed area, although it still showed enough strength to be handled. After the optical tests, samples were cut across the diameter of the bonded pieces and the exposed face polished and observed with SEM. Figure 4 shows sample photographs of the bonded components.
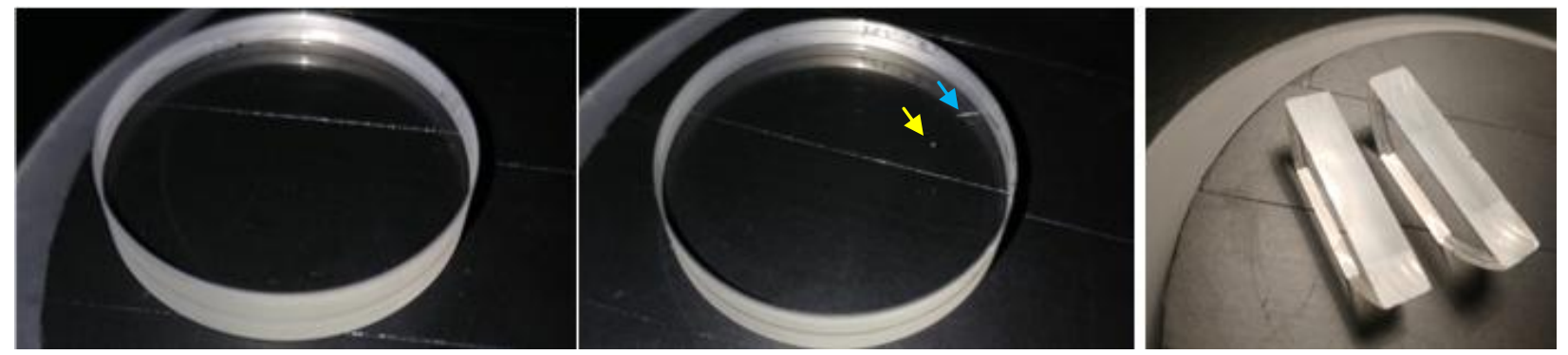

Figure 4: Left: HCB sample. Middle: SB sample with a little spot (yellow arrow) in the bond, the little crack (blue arrow) is on an outer surface. Right: Cut and polished through the bond HCB (left) and SB (right) samples.

There was no noticeable difference between the visually inspected samples before they were put under test. The manufacturing trial also showed no sign of failure and the bond interface can barely be seen anymore after polishing the samples. Figure 5 shows sample pictures taken by SEM. A noticeable difference can be witnessed in the width of the bond line between the HCB and SB samples at the same magnitude. Going to higher magnifications, the resolution degraded significantly due to a non optimal gold coating. However, it was still possible to estimate the bond thicknesses below $50 \mathrm{~nm}$ for the HCB and averaging $120 \mathrm{~nm}$ for the SB along the diameter.
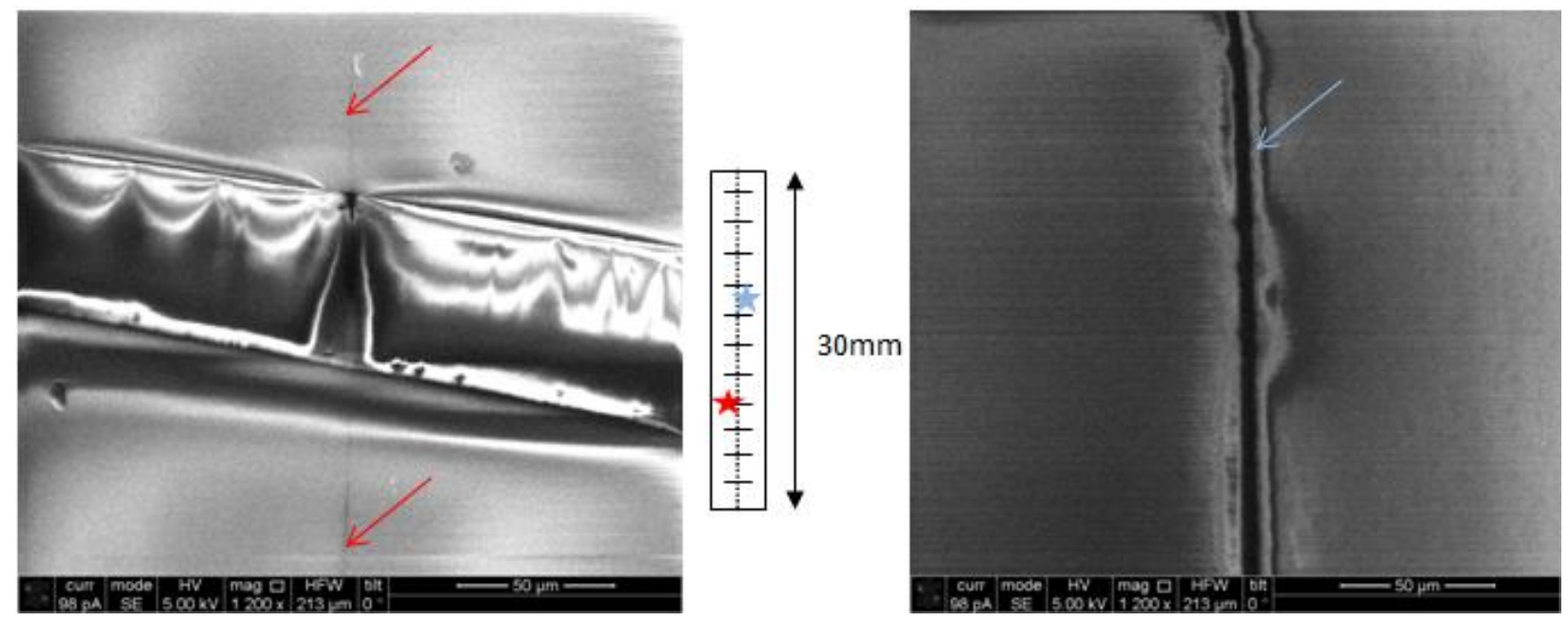

Figure 5: SEM pictures of the HC bond (left) and silicate bond (right) at magnitude x1200. Bond lines are marked with arrows. The central drawing displays the approximate position where the pictures where taken (with the star shifted towards the image it corresponds to). The short lines are cuts made in the coating as a spatial marker as well as a location to take measurmeents throughand the dotted line is the bond line 


\subsection{Light induced damage threshold}

Results from the LIDT tests are displayed in Figure 6.

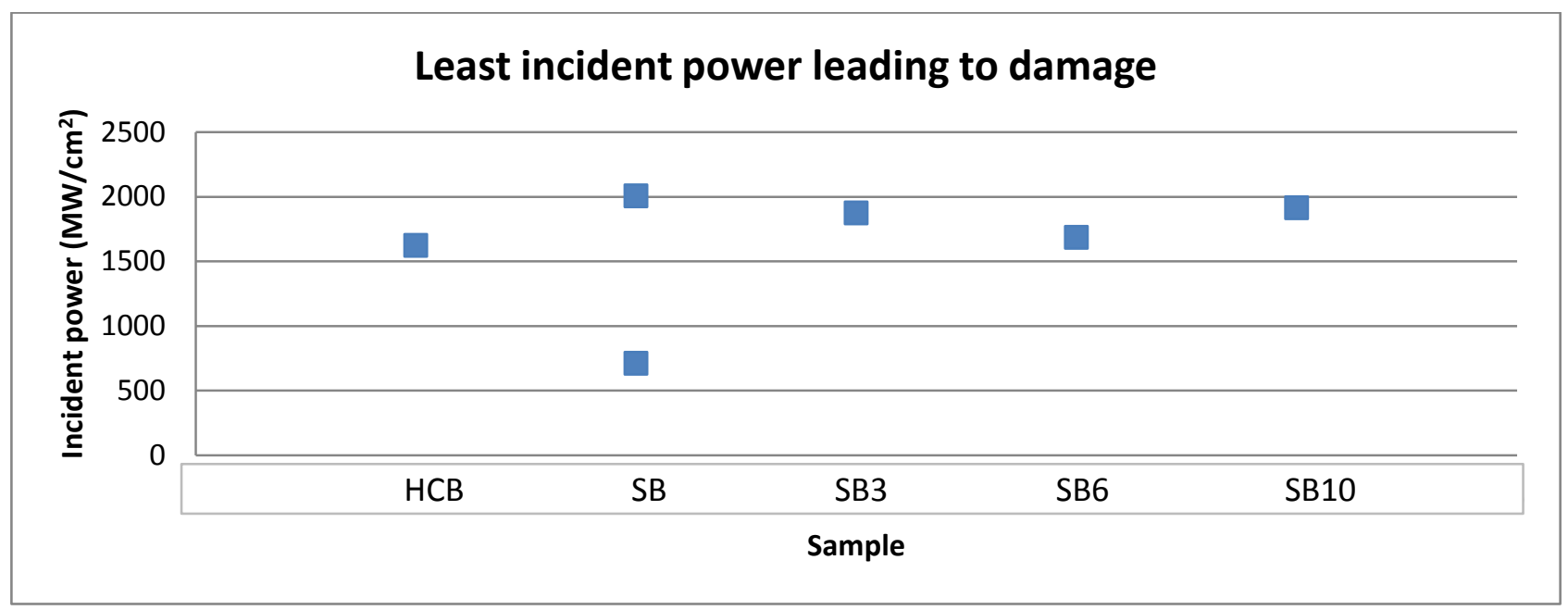

Figure 6: LIDT values for all samples with parameters from Figure 2. HCB and SB measurements are taken after 120 days curing whereas SB3, SB6 and SB10 are taken after 46 days.

For all the samples but SB, a clear threshold could be identified with no damage before the LIDT and consistent damage after. The values measured for the thresholds are $1620 \mathrm{MW} / \mathrm{cm}^{2}, 1870 \mathrm{MW} / \mathrm{cm}^{2}, 1680 \mathrm{MW} / \mathrm{cm}^{2}, 1910 \mathrm{MW} / \mathrm{cm}^{2}$ for HCB, SB3, SB6 and SB10, respectively. Due to the discrete nature of the measurement, the uncertainty is about \pm 50 $\mathrm{MW} / \mathrm{cm}^{2}$. From these values, there seem to be no general trend that would link the solution concentration and the LIDT.

The case of SB was a bit different as the first damage occurred at $710 \mathrm{MW} / \mathrm{cm}^{2}$ which corresponds to the lowest point on the Figure 6. However, when increasing the power several rows showed no damage up to $2000 \mathrm{MW} / \mathrm{cm}^{2}$ (highest point on the Figure 6) from which the bond started to get consistently damaged again. As the subsequent tests on SB3, SB6 and SB10 do not suggest that the threshold is much lower for SB compared to HCB, it is likely that the low value measured is specific to the sample due to inhomogeneities in the bond for example. Therefore the value of 2000 $\mathrm{MW} / \mathrm{cm}^{2}$ is probably more representative of the thresholds that can be obtained with the process. However, the curing times are also different between the sets which prevents from directly comparing them.

\subsection{Reflectivity}

The first set (HCB and SB) was tested after 150 days curing to ensure a steady state was reached. The reflectivity results for HCB and SB samples are shown in Figure 7. It appeared that due to the sample geometry (Ø30 x $3 \mathrm{~mm})$ it was not possible to properly mask the reflections from the surfaces. This limited the sensitivity reachable and proved to be an issue in the case of the HCB sample for which the bond spot was less bright than for its SB counterpart and could not stand out from the background signals due to the peaks from the two surfaces. Solutions to this problem would be to use thicker samples to better separate the three beams, a thinner slit to better isolate the bond spot or applying a treatment to the surfaces to lower their reflection.

As the reflectivity of the sodium hydroxide bond was too low to get a signal from the bond, the curves for this sample are plotted from the values at the middle of the interval and thus only display a ceiling value for the reflectance. The only exception was the $55^{\circ} \mathrm{HCB}$ P-pol for which the surfaces spots are split enough and the bond reflection bright enough to yield a peak. This could give an idea of the offset of the other points as the theoretical curves can be found from the model discussed in section 3.4. 


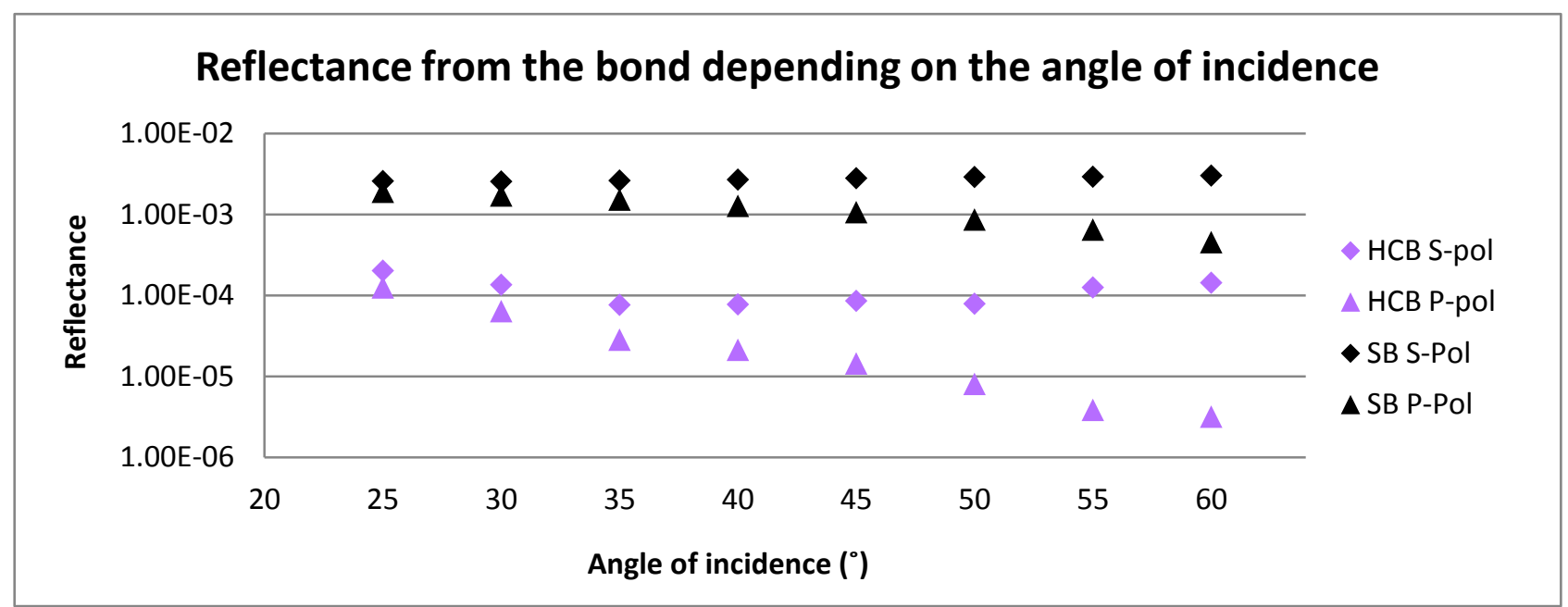

Figure 7: Reflectivity measurements for both HCB and SB (first set of samples), both polarisations when varying the angle of incidence. The HCB curves are only upper limit values of the reflectance as the sample geometry did not allow precise measurements.

In spite of this setback, the measurements still display a noticeable gap between the reflectivity of the two samples. The SB is about ten times more reflective than the HCB with an upper limit value of about $0.3 \%$ (S-pol) and $0.2 \%$ (PPol) for the former and $0.03 \%$ and $0.01 \%$ for the latter. This is expected as the presence of silica yields a bond with a refractive index different from the one of the bulk, whereas the HCB chemistry should be closer to the one of the bulk material.

The second set ( $35 \times 10 \times 5 \mathrm{~mm}$ ) was tested at 3,16 and 28 days of curing with the purpose of monitoring change in reflectivity with curing time. To put the two sets in parallel, HCB could be considered as the extreme case of low concentration of silicate while SB6 should tend to SB with time.

The results are displayed in Figure 8. It can be noticed that a few points are missing. These points were clear outliers, most of which could be tracked back to experimental imprecision. As they would have hindered the general trend they were discarded for the sake of clarity.

The decrease in reflectivity with curing time is noticeable for all three samples and both polarisations as it gets reduced by a factor of 3 or 4 over the month of curing. The value reached after 28 days is between $0.1 \%$ and $0.2 \%$ (Spol) or below $0.1 \%$ (Ppol) for SB3 and SB6 with not much difference between the two samples. Although all cross comparison between the sets have to be made carefully due to several changing parameters, it can be noticed that these values are not very far from the ones obtained with the SB sample presented above which was bonded at 1:6 concentration and measured after being cured to an estimated steady state. In the case of SB10, the reflectivity is overall lower and decreases to about $0.07 \%$ ( $\mathrm{Spol}$ ) and $0.06 \%$ (Ppol), which suggests that it is indeed possible to act on the bond reflectivity through changing the solution concentration. HCB can represent the limit of dilution when there is no silicate added and the reflectivity is indeed even lower than for SB10.

\subsection{Bond properties}

The reflectivity data displayed above were then input in the MATLAB program for each sample at each curing time in order to obtain the curing time depending thickness $\left(\mathrm{d}_{2}\right)$ and refractive index $\left(\mathrm{n}_{2}\right)$ of the bond. An example of the output from the program for the data from SB3 after 16 days of curing is shown in Figure 9. 

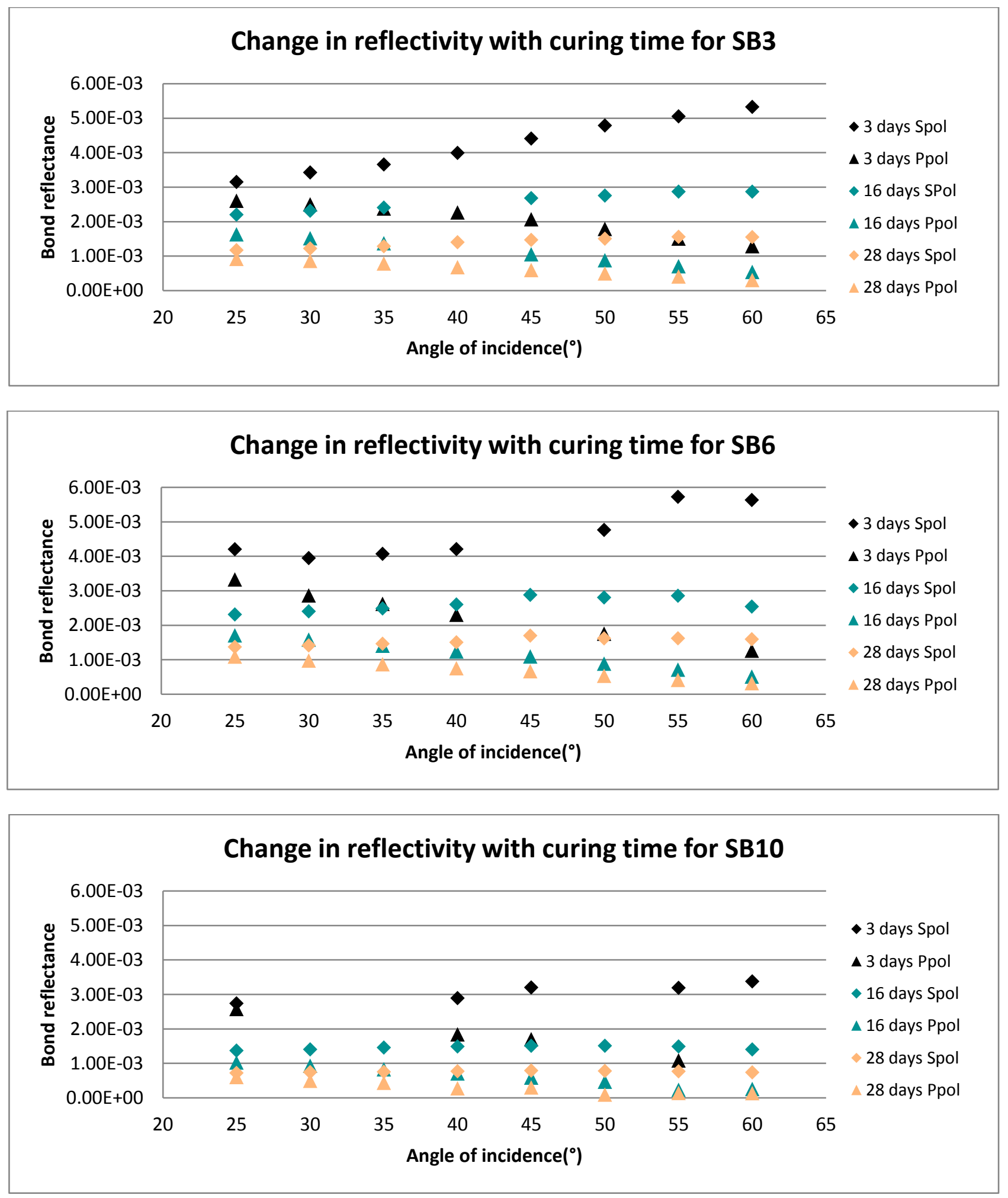

Figure 8: Change of reflectivity with curing time for SB3 (top), SB6 (middle) and SB10 (bottom). From observing the curves of same shape on each graph, a decrease of reflectance over time is noticeable for both polarisation for each sample. SB10 reflectance alsao appears to be lower than SB3 and SB6 at every stage of curing. 

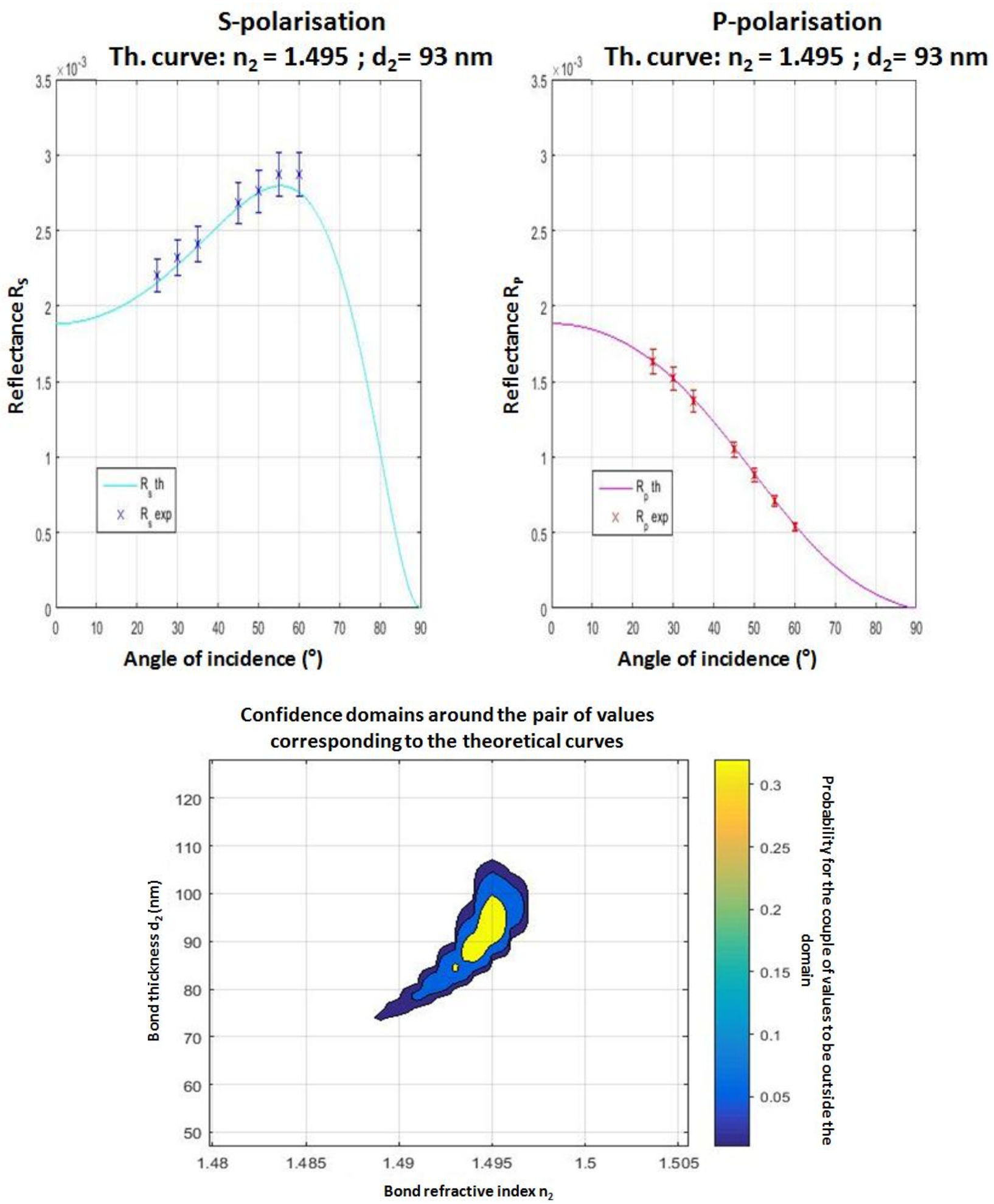

Figure 9: Example of determination of the most probable pair or parameters $n_{2}$ and $d_{2}$ that fits the experimental data for SB3, 16 days curing (top, discrete points). The best fitting theoretical curves for each polarisation (top, continuous lines) are calculated by maximum likelihood Bayesian analysis in the MATLAB script. The bottom plot shows the domains of confidence around the estimated values. 
The program returns the values of thickness and refractive index that allow the best fit with the data. In this case the fit is good and the confidence interval quite narrow. The error is estimated from the confidence interval and will be taken equal to \pm 0.002 for the refractive index and $\pm 7 \mathrm{~nm}$ for the thickness.

By collecting all the outputs it is possible to get the change of these parameters with the curing time, as shown in Figure 10. The results for SB10 are not included as for this particular sample the uncertainty in the determination of parameters was too high to provide reliable results.

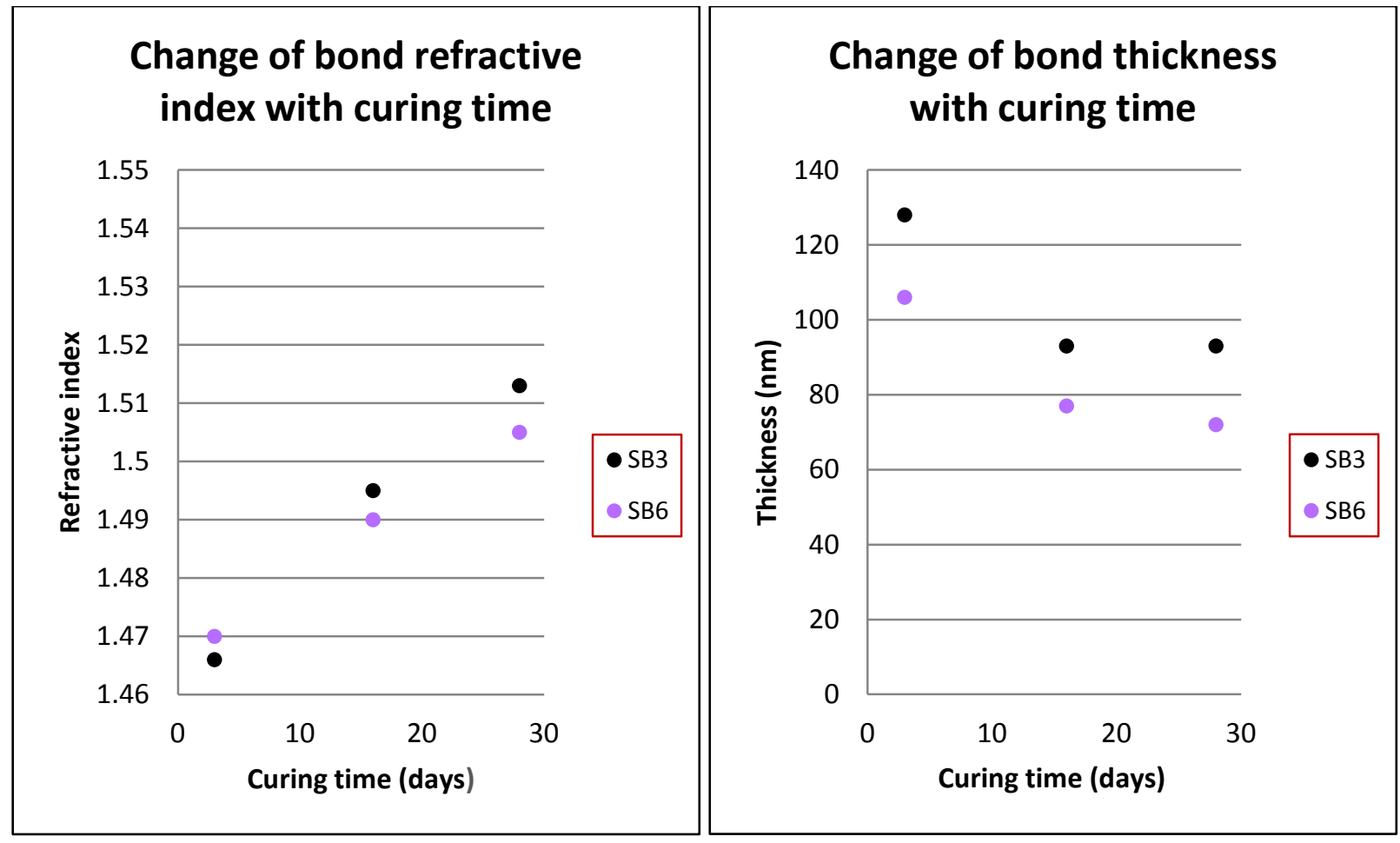

Figure 10: Refractive index (Left) and thickness (right) obtained for SB3 and SB6 at the various stages of curing from the reflectivity data through the MATLAB script.

It can be seen that within the first month of curing, the refractive index increases for both SB3 and SB6, from 1.46 to 1.51 and from 1.47 to 1.50 , respectively. In the meantime the thickness decreases from about 130 to 95 (SB3) and 105 to 70 nanometres (SB6). It seems that over the course of the curing, SB3 has formed a thicker bond with higher refractive index than SB6. It is hard to conclude on the kinetic of the curing with so few points but it seems that the slope decreases rather quickly for the thickness variation while it remains more or less constant for the refractive index variation. It is also worth to notice that the estimated refractive index tends to increase above the one of fused silica (1.48).

\section{DISCUSSION}

The first result of this study is the successful bonding of phosphate glass with the HCB technique. Using a sodium silicate solution, it is expected to be able to bond most oxides ${ }^{18}$ and the present work reinforces this hypothesis. However a more significant advance is the possibility to make bonds with a simple hydroxide solution. Although the chemistry has not been thoroughly investigated, the bond appears free of defects and the sample can be handled and manufactured proving a relevant strength. A similar process is patented by Schott AG but is limited to bonding phosphate glass using an aqueous phosphorous solution ${ }^{19}$. 
Being able to cut and polish the samples through the bond allowed observation of the bond line under SEM. It is not the first time it has been done and estimations of silicate bonds thickness from direct observation usually lie between $40 \mathrm{~nm}$ and $100 \mathrm{~nm}$ for bonds between materials such as fused silica or oxidised silicon, with various bonding parameters $^{19}$. The values observed in this work are in the same range which is not too surprising given the similarities in surface preparation. It also appears that the bond thickness changes significantly with the concentration of the sodium silicate solution used. In the first set, SEM observations show HCB and SB samples with bond thickness below $50 \mathrm{~nm}$ and around $120 \mathrm{~nm}$, respectively. The difference can be explained as silicate bonding brings more solid constituents to the network than HCB which essentially only etches the surfaces. The derived thickness from the reflectivity measurements seem to confirm this result as the more concentrated solution gives a thicker bond (by about 20 nanometres) at each stage probed during the curing. The thicknesses of SB3 and SB6 end up being around 90 and 70 nanometres respectively, which are values in the expected range.

The LIDT tests at $1535 \mathrm{~nm}$ gave a value between $1620 \mathrm{MW} / \mathrm{cm}^{2}$ and $1910 \mathrm{MW} / \mathrm{cm}^{2}$ for all samples but for SB, the case of which has already been discussed in section 4.1. No previous data has been found for tests on bonds for the same material but LIDT tests have been carried on fused silica bonded with the same technique. Beveridge ${ }^{11}$ reports LIDT in a range from 300 to $860 \mathrm{MW} / \mathrm{cm}^{2}$ for fused silica bonds at $1064 \mathrm{~nm}$ while $\mathrm{Sinha}^{8}$ recorded values as high as 2200 $\mathrm{MW} / \mathrm{cm}^{2}$ for similar samples and parameters. Despite the different wavelength and materials, all the thresholds reported in these studies alongside the ones of this work suggest that the LIDT of the bond is well above what is required for common applications in photonics and high power lasers. The results from this work do not allow any highlight of the influence of the concentration on the power at which the first damage is witnessed as all thresholds measured are in the same range. This contrasts with Sinha's work which reports that when bonding fused silica, a higher concentration of the sodium silicate solution lead to a higher damage threshold. This could be due to the difference in chemistry or only to the low amount of samples tested coupled with the uncertainty of the test.

The reflectivity measurements showed a reflectance of the order of magnitude of $0.1 \%$ (-30dB) for SB and $0.01 \%$ ($40 \mathrm{~dB}$ ) for HCB although accurate measurements were not possible in the case of the latter. Sinha's work ${ }^{8}$ provides values to compare with despite using fused silica and a different wavelength $(1064 \mathrm{~nm})$ and setup layout. A notable difference is that he measured the feedback at normal incidence, using temporal separation rather than spatial separation of the signals from the different interfaces. However the model based on thin layer interferences used in Section 4.3 suggests that the reflectivity at normal incidence would be close in value to the ones in the range investigated in this work (see incidence at $0^{\circ}$ on Figure 8).

Sinha measured a reflectance from the bond of $-55 \mathrm{~dB}$ for samples prepared in the closest conditions to the ones of this work. This means that the reflectivity of the bond is significantly lower than the ones measured here (10 or 100 times depending on the sample). The natural explanation is that the silicate solution matches better the index of fused silica than the one of phosphate glass (at the respective wavelengths at which the measurements are made) but also helps building a denser network than what can achieve the simple hydroxide solution, and thus decrease the reflection due to micro-voids in the bond.

As the purpose is to minimise the reflectance from the bond, the HCB technique seems to perform better than the SB one. Usual requirements for surfaces and interfaces reflectivity in high power laser components are to be below $0.2 \%$ which is met by a 10 times margin with the HCB and not exactly met by the SB method. Sinha showed that increasing the volume of solution or the silicate concentration helps decreasing the feedback from the bond by increasing the density of the network (amount of silicate at the interface). However the results presented in Section 4.2 show that these conclusions do not transfer to the case of phosphate glass as the solution diluted 11 times (SB10) yields a lower reflectivity than the solutions diluted 4 (SB3) and 7 (SB6) times. It can be understood as a denser layer with a refractive index different from the one of the bulk hardly helps reducing the reflectivity of the interface. 
The estimation of the refractive index from the reflectivity measurement suggests that the refractive index of the layer after a few days curing is between the one of fused silica (1.48) and the one of phosphate glass (1.56). It was not the case with fused silica or sapphire for which the index of the layer peaked at $1.48^{9}$. It means that contrary to sapphire, phosphate glass gets etched by the solution and some phosphate chains enter the bond yielding a higher refractive index than if it was pure fused silica. Therefore there are two expected consequences from increasing the concentration of sodium silicate solution. On the one hand there are more silicate ions added to the network which should bring the refractive index of the layer down and increase the mismatch with the bulk and hence, the reflectivity. On the other hand, the concentration of hydroxide ions likely increases the amount of phosphate chains that will go in the bond and bring the refractive index up. As there is no significant difference in reflectivity between SB3 and SB6 but there is between them and SB10, there may be a threshold when one of the effects takes over the other and it may be possible to find an optimal concentration to minimise the reflectivity.

In the end, HCB seems to fulfil the requirements to bond phosphate glass components better than all the silicate bonded ones. However, the main reason to use sodium silicate over sodium hydroxide has been the added mechanical strength and although photonics components should not have to bear the same stresses as large glass assemblies, they could still be subject to vibration or shock for example during the transportation phases. Mechanical strength is also an asset to allow the manufacturing of bonded components and quantitative strength test trials are currently in preparation. If it appears that the silicate bond is significantly stronger than the HCB, it will be relevant to use the results from this work to find a compromise between strength and optical properties by changing the concentration of the sodium silicate solution.

\section{CONCLUSION}

We successfully bonded phosphate glass using Hydroxide Catalysed Bonding (HCB), opening new potential applications for a method that has shown its reliability throughout the past years. Measurements of the reflectivity and Light Induced Damage Threshold of the bonds showed that both these properties met the usual requirements for high power laser components. All these results go in a favourable direction regarding the possibility of transfer of the technique from the mechanical to the optical field. HCB would join the already used bonding techniques of the field and gives more options to choose the more adapted one, particularly the opportunity to bond at room temperature and therefore limit the thermal residual stresses in bonded components.

\section{ACKNOWLEDGEMENTS}

We would like to thank Belford Research for carrying out the LIDT measurements and Jamie Scott for his assistance in the SEM observations. The research leading to these results has received funding from the People Programme (Marie Curie Actions) of the European Union's Seventh Framework Programme FP7/2007-2013/ (PEOPLE-2013-ITN) under REA grant agreement $n^{\circ}$ [606176]. It reflects only the authors' view and the Union is not liable for any use that may be made of the information contained therein. Mariëlle van Veggel is supported by an RS Dorothy Hodgkin Fellowship [DH120021] and the reflectivity set-up was developed with support from the RS through Research grant, PI Marielle van Veggel [RG120367,] that supported PGR student Valentina Mangano. 


\section{REFERENCES}

[1] Gwo DH. , "Two unique aspects of gravity probe-B star-tracking space telescope: (1) focal-plane roof-edge diffraction and (2) fused-quartz bonding for 2.5-Kelvin applications" Proc. SPIE 3356, Space Telescopes and Instruments V, 892 (April 11, 1998).

[2] Chang Y. T., Huang Y., Su K. W. and Chen Y.F., "Comparison of thermal lensing effects between single-end and double-end diffusion-bonded Nd:YVO4 crystals for $4 \mathrm{~F} 3 / 2 \rightarrow 4 \mathrm{I} 11 / 2$ and $4 \mathrm{~F} 3 / 2 \rightarrow 4 \mathrm{I} 13 / 2$ transitions", Optics express, 16(25), 21155-21160 (2008).

[3] Haisma J., "Contact bonding, including direct bonding in a historical and recent context of materials science and technology, physics and chemistry", Materials Science and Engineering, R(37), 1-60 (2002).

[4] Lee H., Brownlie P.L., Meissner H.E. and Rea E.C., "Diffusion Bonded composites of YAG single crystals", Proc. SPIE 1624, Laser-Induced Damage in Optical Materials: 1991, (29 July 1992).

[5] Dillard D.A., [Advances in structural adhesive bonding], Boca Raton : Woodhead publishing limited, 1-35 (2010).

[6] Knetchel R., "Glass frit bonding: An universal technology for wafer level encapsulation and packaging", Microsystem technologies, 12(1), 63-68 (2005).

[7] Van Veggel A.A. and Killow C.J., "Hydroxide catalysed bonding for astronomical instruments", Advances in optical technologies, 3(3), 293-307 (2014).

[8] Sinha S., Urbanek K.E., Krzywicki A. and Byer R.L., "Investigation of the suitability of silicate bonding for facet termination in active fiber device", Optics express, 15(20), 13003-130022 (2007).

[9] Mangano V., Van Veggel A.A., Douglas R., Faller J., Grant A., Hough J., Rowan S.,"Determination of the refractive index and thickness of a hydroxide-catalysis bond between fused silica from reflectivity measurements", Optics express, 25(4), 3196-3214 (2017).

[10] Taylor A., [Aspects of Optical Metrology systems for Space-Borne Gravitational Wave Detector], Glasgow, Glasgow theses service, Chapter 3, 16-44 (2014).

[11] Beveridge N., "KTP on Adhesive free bonding," Unpublished document Gooch and Housego and University of Glasgow, Ilminster, (2012).

[12] Hayden J.S., Hayden Y.T., Campbell J.H., "Effect of composition on the thermal, mechanical and optical properties of phosphate laser glasses", Proc. SPIE 1277, High-Power Solid State Lasers and Applications (1990).

[13] Li W., He D., Li S., Chen W., Chen S., Hu L., "Optical and thermal properties of a New Nd-doped phosphate laser glass,” Proc. SPIE 8786, Pacific Rim Laser Damage 2013: Optical Materials for High Power Lasers, 878629 (July 9, 2013).

[14] Douglas R., Van Veggel A.A., Cunningham L., Haughian K., Hough J., Rowan S., "Cryogenic and room temperature strength of sapphire jointed by hydroxide-catalysis bonding", Classical and Quantum Gravity, 31(4), 45001-45011 (2014).

[15] Bunker B.C., Arnold G.W., Wilder J.A., "Phosphate glass dissolution in aqueous solution”, Journal of NonCrystalline Solids, 64(3), 291-316 (1984).

[16] Manenkov A.A., "Fundamental mechanisms of laser-induced damage in optical materials: today's state of understanding and problems", Optical engineering, 53(1), 10901 (2014). 
[17] Papernov S., Schmid A.W., "Laser-induced surface damage of optical materials: absorption sources, initiation, growth, and mitigation”, Proc. SPIE 7132, Laser-Induced Damage in Optical Materials: 2008, 71321J (30 December 2008).

[18] Gwo, "US Patent 6548176 B1: Hydroxide Catalyzed bonding", 2003.

[19] Schott Glass Technologies Inc., "US Patent 6652972: Low temperature joining of phosphate glass”, 2003.

[20] Elliffe E.J., Bogenstahl J., Deshpande A., Hough J., Killow C.J., Reid S., Robertson D., Rowan S., Ward H., Cagnoli G. "Hydroxide-catalysis bonding for stable optical systems for space", Classical and Quantum Gravity, 22(10), 257-267(2005). 